\title{
Investigação da reatividade e da cinética de dissolução do metacaulim em ácido sulfúrico
}

\author{
(Investigation of reactivity and dissolution kinetics of \\ metakaolin in sulfuric acid) \\ P. E. A. de Lima ${ }^{1}$, R. S. Angélica ${ }^{1}$, T. Scheller ${ }^{1}$, R. F. Neves ${ }^{2}$ \\ ${ }^{I}$ Programa de Pós-Graduação em Geologia e Geoquímica, Instituto de Geociências (PPGG-IG), \\ ${ }^{2}$ Programa de Pós-Graduação em Engenharia Química, Faculdade de Engenharia Química (PPEQ-FEQ) \\ Universidade Federal do Pará, R. Augusto Corrêa s/n, Belém, PA 66075-110 \\ paulalima@ufpa.br
}

\begin{abstract}
Resumo
Um estudo da reatividade e da cinética de dissolução do metacaulim foi desenvolvido para o caulim de cobertura de papel oriundo da região do Rio Capim, Pará. O caulim foi calcinado a $600,700,800,900$ e $1000{ }^{\circ} \mathrm{C}$ por $2 \mathrm{~h}$ para obtenção do metacaulim e posteriormente lixiviado com ácido sulfúrico em temperaturas constantes dentro do intervalo $50-95{ }^{\circ} \mathrm{C} \pm 3{ }^{\circ} \mathrm{C}$. Nas lixiviações foram usadas quantidades de ácido sulfúrico correspondentes a $10 \%$ acima do valor estequiométrico em relação ao alumínio presente no material. Foram coletadas amostras, com intervalos pré-determinados a cada $15 \mathrm{~min}$ até tempo total de $3 \mathrm{~h}$, as quais foram submetidas à análise de teor de alumínio pelo método titrimétrico com EDTA. Os resultados mostraram que a reatividade do caulim calcinado diminuiu com o aumento da temperatura de calcinação e que a cinética de dissolução do alumínio do metacaulim obtido por calcinação a $700{ }^{\circ} \mathrm{C}$ seguiu os métodos de reação homogênea utilizados: método integral, método das meia-vidas e método das velocidades iniciais. Uma energia de ativação de $96,25 \mathrm{~kJ} / \mathrm{mol}$ foi encontrada bem como reação de lixiviação de primeira ordem em relação ao alumínio do metacaulim e de primeira ordem em relação à concentração ácida. Pesquisas realizadas anteriormente, utilizando excesso de um dos reagentes acima de $90 \%$ e modelos de reação heterogênea, estão em consonância com os dados encontrados neste trabalho desenvolvido com excesso de apenas $10 \%$.
\end{abstract}

Palavras-chave: caulim, metacaulim, lixiviação, reatividade.

\begin{abstract}
A study about reactivity and dissolution kinetics of metakaolin was carried out using kaolin for paper coating derived from the Rio Capim region, Pará state, northern Brazil. The kaolin was burned at 600, 700, 800, 900 and $1000^{\circ} \mathrm{C}$ during $2 \mathrm{~h}$ in order to obtain metakaolin, followed by leaching with sulfuric acid at constant temperatures within the $50-95{ }^{\circ} \mathrm{C} \pm 3{ }^{\circ} \mathrm{C}$ range. In the leachings, amounts of sulfuric acid corresponding to $10 \%$ above the stoichiometric values were used with respect to aluminum present in the material. Samples were collected in predetermined intervals every 15 min until a total time of $3 \mathrm{~h}$, which were subjected to aluminum analysis by the EDTA titrimetric method. The results showed that the reactivity of burned kaolin decreased with the increase of burning temperature and that the dissolution kinetics of aluminum from metakaolin obtained by burning at $700{ }^{\circ} \mathrm{C}$ followed the methods of homogeneous reaction used: integral method, method of half-lives and method of initial rates. $96.25 \mathrm{~kJ} / \mathrm{mol}$ of activation energy was found as well as leaching reaction of the first order with respect to aluminum from metakaolin and the first order with respect to acid concentration. Researches carried out previously, using one of the reagents with excess above $90 \%$ and models of heterogeneous reaction, are consistent with the data found in this paper developed with only $10 \%$ excess.
\end{abstract}

Keywords: kaolin, metakaolin, leaching, reactivity.

\section{INTRODUÇÃO}

Na região amazônica encontram-se grandes e valiosas reservas de bauxita gibsítica e de caulim, as quais consistem nas duas principais fontes de alumínio. As três principais minas de caulim são uma mina nas proximidades do Rio Jari, Amapá, sendo atualmente explorada pela empresa Caulim da Amazônia S.A. (CADAM) e duas minas na região do Rio Capim, nordeste do Estado do Pará, com o processo de beneficiamento realizado pela empresa Imerys Rio Capim Caulim S.A. (IRCC). As minas citadas apresentam caulim com alto grau de alvura para indústria de cobertura de papel e baixo teor de contaminantes [1].

O processo de beneficiamento do caulim para cobertura gera um elevado volume de rejeito poluente que permanece em lagoas de sedimentação para sua deposição. Com isso, constitui um problema ambiental visto que necessita de longo tempo para sedimentação até apresentar consistência que possibilite o revestimento para recuperação da área, em contrapartida, apresenta elevado nível de pureza e é superior aos caulins do Sul e Sudeste do Brasil. O rejeito, constituído essencialmente de caulinita, consiste em um material com 
potencial para ser usado em áreas como construção civil e cerâmica [2-5].

O principal método para obtenção de alumínio baseiase no processo Bayer, que utiliza solução básica e bauxita como fonte de matéria-prima. No entanto, existem fontes alternativas de alumínio via processo ácido, como o caulim presente em abundância na região amazônica. Neste procedimento, os principais ácidos utilizados são ácido sulfúrico, clorídrico e nítrico [6]. Para promover a extração do alumínio do caulim através do processo ácido, primeiramente é necessário efetuar a calcinação do material para transformação da caulinita $\left(\mathrm{Al}_{2} \mathrm{Si}_{2} \mathrm{O}_{5}(\mathrm{OH})_{4}\right)$ em metacaulim $\left(\mathrm{Al}_{2} \mathrm{Si}_{2} \mathrm{O}_{7}\right)$, um composto amorfo e altamente reativo obtido pela desidroxilação da caulinita. A reatividade do metacaulim depende fortemente da temperatura na qual ocorreu a sua obtenção e os efeitos da calcinação na solubilidade do alumínio não são claramente entendidos. Ford [7] relatou que Grim em 1968 e Barclay e Peters em 1976 sugeriram que a solubilidade melhorada do alumínio no caulim calcinado surge a partir da ruptura da caulinita na qual cátions de alumínio são convertidos da coordenação octaédrica para tetraédrica com ânions de oxigênio. Phillips e Wills [8] afirmaram que a caulinita pode ser convertida em metacaulim pelo aquecimento na faixa de temperatura $473-900{ }^{\circ} \mathrm{C}$ e que o alumínio pode ser extraído muito mais facilmente do metacaulim do que da caulinita. Segundo Gajam e Raghavan [9], a opinião geral é que a calcinação proporciona o aumento da reatividade das partículas além de promover o processo de desidroxilação e a remoção de compostos orgânicos presentes. Belver, Muñoz e Vicente [10] concluíram que o metacaulim obtido por calcinação a $900^{\circ} \mathrm{C}$ é menos reativo do que o obtido a 600,700 e $800{ }^{\circ} \mathrm{C}$ e explicaram que tal fato se deve à sinterização incipiente a 900 ${ }^{\circ} \mathrm{C}$. A influência da calcinação na reatividade do metacaulim foi e tem sido discutida por diversos autores, entretanto este processo ainda não foi demonstrado numericamente e o que acontece com o metacaulim ainda é um tema em estudo, visto que do ponto de vista da difração de raios $\mathrm{X}$ é apenas um material amorfo.

Alguns pesquisadores também já realizaram trabalhos acerca da cinética de dissolução do alumínio do metacaulim. Em 1970, foi discutida a cinética da alumina removida do caulim calcinado com ácido nítrico, sulfúrico e clorídrico [6]. Em 1985 foi apresentado um modelo cinético para lixiviação da argila caulinítica com ácido clorídrico na presença de íons fluoreto [9]. Em 1992 foi estudada a lixiviação de um caulim oriundo da África com ácido sulfúrico [7]. Em 2003 foi investigada a cinética de dissolução do caulim com ácido clorídrico [11]. Em 2010 foi estudada a cinética de dissolução do metacaulim com ácido sulfúrico, dentre outros [12]. A maioria dessas pesquisas foi desenvolvida com a utilização de caulins de referência, com excesso de reagente muito elevado, acima de $90 \%$, e com a aplicação de métodos de reação heterogênea. Assim, os principais pontos que justificam a realização do trabalho são: a) estudos cinéticos de dissolução do metacaulim ainda não foram desenvolvidos para caulins da região amazônica; b) a utilização de um excesso elevado de um dos reagentes envolvidos, apesar de ser recomendada pela literatura, não é industrialmente viável devido a fatores econômicos; e c) os trabalhos realizados sobre a dissolução do metacaulim utilizam somente métodos de reação heterogênea e não aplicam métodos de reação homogênea afim de comparação entre as duas abordagens.

O objetivo do presente trabalho consiste em desenvolver um estudo da reatividade do metacaulim através do processo de desaluminação promovido por lixiviação com ácido sulfúrico e realizar um levantamento cinético desse processo com uma abordagem de reação homogênea e a heterogênea estando em execução para ser apresentada posteriormente. Para isso, foi utilizado um caulim de cobertura amazônico, processado, e ácido sulfúrico com $10 \%$ acima do seu valor estequiométrico.

\section{MATERIAIS E MÉTODOS}

Como material de partida foi utilizado o caulim para cobertura de papel da região do Rio Capim, Pará, e o ácido sulfúrico, com pureza $95-98 \%$, em concentrações que proporcionassem as relações alumínio/ácido iguais à estequiometria de reação com $10 \%$ de excesso. O caulim foi inicialmente seco em estufa a $110^{\circ} \mathrm{C}$, em seguida calcinado em forno mufla a $600,700,800,900$ e $1000^{\circ} \mathrm{C}$ por $2 \mathrm{~h}$ e as amostras resultantes das calcinações receberam as seguintes denominações: M600, M700, M800, M900 e M1000, respectivamente.

Caracterização dos materiais: os dados difratométricos do caulim, antes e depois das calcinações, foram obtidos pelo método do pó em difratômetro de raios X PANalytical X'Pert Pro MPD (PW 3040/60) com goniômetro PW 3050/60 $(\theta-\theta)$, com tubo de raios X cerâmico de anodo de $\mathrm{Cu}\left(\mathrm{k} \alpha_{1}=1,54060 \AA\right), \mathrm{PW} 3373 / 00$ e foco fino longo (2200 $\mathrm{W}-60 \mathrm{kV}$ ). Foi utilizado filtro $\mathrm{k} \beta$ de $\mathrm{Ni}$, ângulos de varredura de $4^{\circ}$ a $75^{\circ}[2 \theta]$, voltagem $40 \mathrm{kV}$, corrente $30 \mathrm{~mA}$, tamanho do passo $0,017^{\circ}$ [20], tempo/passo de $20 \mathrm{~s}$, fenda divergente de $1 / 4^{\circ}$, anti-espalhamento de $1 / 2^{\circ}$ e máscara de $10 \mathrm{~mm}$. Para complementação da análise difratométrica foi realizado estudo in situ por DRX. Esse método trata de um procedimento térmico no qual o aquecimento do material é realizado sobre uma lâmina de platina no próprio equipamento e simultaneamente são realizadas análises difratométricas. $\mathrm{O}$ estudo da transformação térmica foi realizado nas temperaturas de $970{ }^{\circ} \mathrm{C}$ e $1100{ }^{\circ} \mathrm{C}$, nos tempos de $15,30,45,60$ e 120 min e com um ângulo de varredura de 10 a $38^{\circ}$ [2 $\theta$ ]. Foi efetuado o refinamento pelo método de Rietveld da caulinita, com a consequente análise quantitativa das fases, através do software Fullprof [13].

A análise química das amostras foi feita com disco fundido ( $1 \mathrm{~g}$ de amostra $+6 \mathrm{~g}$ de $\mathrm{Li}_{2} \mathrm{~B}_{4} \mathrm{O}_{7}$ ) por espectrometria de fluorescência de raios $\mathrm{X}$ (FRX) em equipamento AxiosMinerals PANAlytical, com tubo de raios X cerâmico, anodo de Rh, máximo nível de potência $2,4 \mathrm{~kW}$ e utilizando o programa IQ+. A perda ao fogo foi determinada por 
diferença de massas após calcinação das amostras a $1000{ }^{\circ} \mathrm{C}$.

A análise granulométrica foi feita em granulômetro a laser Analysette 22 MicroTec Plus Fritsch, a análise da área específica (BET) em Micromeritics TriStar II 3020 e a microscopia eletrônica de varredura (MEV) em equipamento Zeiss LEO 1430.

Lixiviação ácida: o processo de lixiviação ácida foi realizado para o caulim de cobertura não calcinado e para o caulim calcinado nas diferentes temperaturas anteriormente descritas. Os testes padronizados das lixiviações foram desenvolvidos com tempo de residência de $3 \mathrm{~h}$, com $50 \mathrm{~g}$ da amostra inicial e ácido sulfúrico $10 \%$ acima do seu valor estequiométrico, de acordo com a reação:

$$
\mathrm{H}_{2} \mathrm{SO}_{4}+1 / 3 \mathrm{Al}_{2} \mathrm{O}_{3} \rightarrow 1 / 3 \mathrm{Al}_{2}\left(\mathrm{SO}_{4}\right)_{3+} \mathrm{H}_{2} \mathrm{O}
$$

O M700 foi lixiviado a $95,80,70$ e $50{ }^{\circ} \mathrm{C} \pm 3{ }^{\circ} \mathrm{C}$ e o restante das amostras a $95{ }^{\circ} \mathrm{C} \pm 3{ }^{\circ} \mathrm{C}$. Alíquotas foram coletadas a cada 15 min e imediatamente filtradas. Os passantes, livres de sólidos e resfriados, foram submetidos à análise de alumínio pelo método titrimétrico com EDTA.

As lixiviações foram desenvolvidas em um reator com capacidade de $1000 \mathrm{~mL}$ em manta de aquecimento, sob constante agitação e sob refluxo, com termômetro e termostato acoplados. As reatividades ou extrações de alumínio foram analisadas por meio do cálculo das conversões fracionais $(\mathrm{X})$ a fim de acompanhar o progresso das reações, de acordo com a equação:

$$
\mathrm{X}=\frac{\mathrm{n}_{0}-\mathrm{n}}{\mathrm{n}_{0}}
$$

$\mathrm{n}_{\mathrm{o}}$ e $\mathrm{n}$ representam o número de mol inicial e final do reagente limitante, respectivamente. Os ajustes matemáticos dos dados obtidos foram efetuados no programa Microsoft Excel, ferramenta Solver, equação da hipérbole. Todas as lixiviações foram realizadas em duplicata e o valor considerado no trabalho corresponde à média aritmética dos resultados obtidos nos ajustes.

Estudo cinético: o estudo cinético foi feito pelos métodos de reação homogênea, segundo a consideração de que o tamanho das partículas de caulinita é extremamente baixo $(<2 \mu \mathrm{m})$. Foram utilizados os seguintes métodos: integral, das meias-vidas e das velocidades iniciais.

Método integral: utilizado para determinar a ordem da reação através da integração de uma equação diferencial usada para modelar o sistema em batelada. A consistência da equação é avaliada pelos seguintes procedimentos gráficos: a) concentração em função do tempo (ordem zero); b) ln (concentração inicial/concentração) em função do tempo (primeira ordem); e c) 1/(concentração) em função do tempo (segunda ordem). A sua aplicação foi realizada segundo o alumínio dissolvido, foi considerado um intervalo de tempo total necessário para converter $50 \%$ do reagente limitante e o cálculo das constantes de velocidades foi efetuado através do coeficiente angular da reta ajustada do gráfico que se aplicou ao caso em questão $[14,15]$.

Método das meias-vidas: a meia-vida de uma reação, $t_{1 / 2}$, consiste no tempo necessário para que a concentração do reagente seja reduzida à metade do seu valor inicial, ou seja, conversão de $50 \%$. Pode ser visto como um indicador da velocidade com que um reagente é consumido em uma reação química, ou seja, quanto maior for a meia-vida, mais lenta será a reação [14-16].

Método das velocidades iniciais: trata-se de um procedimento diferencial de análise de dados com boa precisão, a partir do qual se determina a expressão de velocidade de reação [14]. Para aplicação do método foram realizadas lixiviações do $\mathrm{M} 700 \mathrm{com}_{2} \mathrm{SO}_{4}$ a $95{ }^{\circ} \mathrm{C} \pm 3{ }^{\circ} \mathrm{C}$. A concentração inicial do ácido foi mantida constante $(1,5 \mathrm{M})$ e variou-se a do alumínio $(0,21,0,43$ e $0,85 \mathrm{M})$, com o objetivo de avaliar o comportamento da sua velocidade de dissolução. Amostras foram coletadas com 15, 20, 25 e $30 \mathrm{~min}$ de reação. Os experimentos foram realizados em duplicata e os ajustes matemáticos foram efetuados no programa Microsoft Excel, ferramenta Solver, equação da hipérbole. $\mathrm{O}$ valor considerado no trabalho corresponde à média aritmética dos resultados obtidos nos ajustes.

Energia de ativação: a energia de ativação é o valor mínimo de energia que as moléculas dos reagentes devem possuir para formação dos produtos. Para o seu cálculo foi utilizada a equação de Arrhenius nas temperaturas de 70, 80 e $95{ }^{\circ} \mathrm{C} \pm 3{ }^{\circ} \mathrm{C}$, considerando o mesmo consumo do reagente limitante usado para o método integral [15].

\section{RESULTADOS E DISCUSSÃO}

\section{Caracterização dos materiais}

Na Fig. 1 encontram-se os difratogramas de raios $\mathrm{X}$ do caulim e do metacaulim obtido por calcinação a 600 , 700, 800, 900 e $1000{ }^{\circ} \mathrm{C}$ (M600, M700, M800, M900 e M1000, respectivamente), durante $2 \mathrm{~h}$. Por meio da análise de difração de raios $\mathrm{X}$ do caulim observou-se que a fase predominante foi a caulinita com a presença do mineral acessório anatásio. No refinamento pelo método de Rietveld verificou-se que a amostra era constituída de 99,59\% de caulinita e $0,41 \%$ de anatásio. A composição química do caulim (Tabela I) confirmou a presença do anatásio com um teor de $0,39 \%$. A partir desses resultados, a amostra pode ser considerada caulinita pura e servir como material de referência. A identificação da caulinita foi confirmada com o arquivo PDF 78-1996 e do anatásio com o arquivo PDF 71-1166.

O processo de calcinação para obtenção do M600 por $2 \mathrm{~h}$ não conseguiu destruir completamente a estrutura da caulinita, para tal seria necessário mais tempo. Assim, este material não foi utilizado nos trabalhos seguintes de 


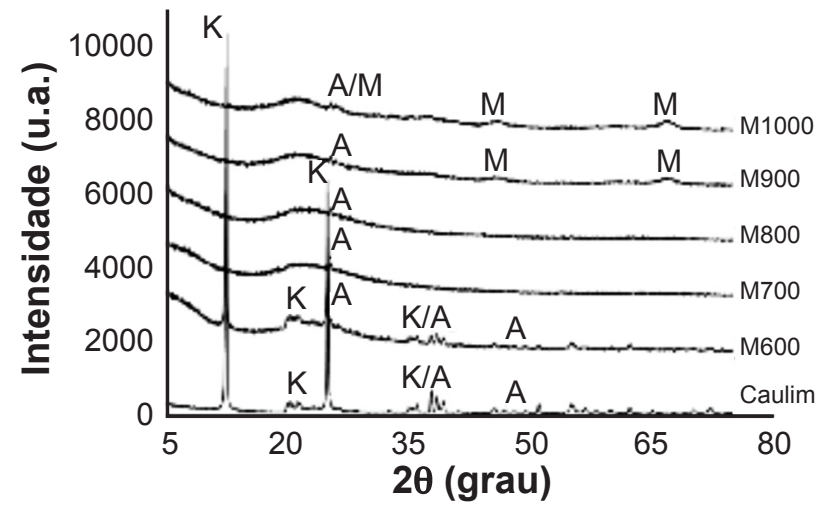

Figura 1: Difratogramas de raios $\mathrm{X}$ do caulim e metacaulim (K: aulinita, A: anatásio e M: mullita).

[Figure 1: X-ray diffraction patterns of kaolin and metakaolin (K: kaolinite, A: anatase and M: mullite).]

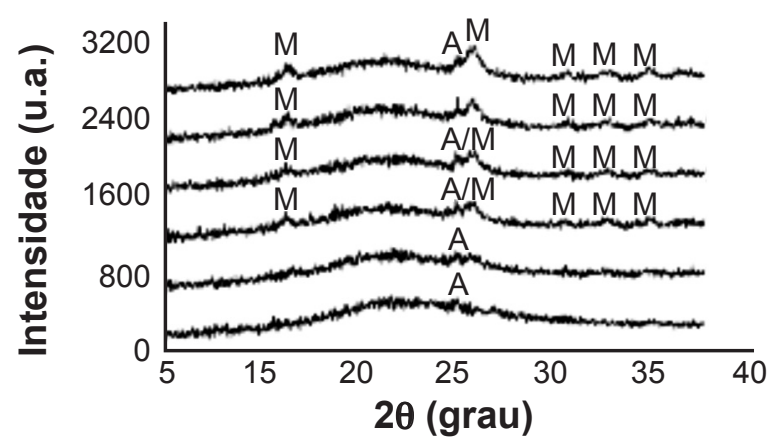

Figura 2: Difratogramas de raios $\mathrm{X}$ obtidos por análise in situ do caulim a $970{ }^{\circ} \mathrm{C}$ nos tempos de $15,30,45,60$ e 120 min e $1100{ }^{\circ} \mathrm{C}$ a 30 min. (M: mullita e A: anatásio).

[Figure 2: X-ray diffraction patterns obtained by in situ analysis of kaolin at $970^{\circ} \mathrm{C}$ in times of $15,30,45,60$ and 120 min and $1100{ }^{\circ} \mathrm{C}$ at $30 \mathrm{~min}$. (M: mullite and A: anatase).]

lixiviação e cinética. O M700 e o M800 indicaram a formação do metacaulim. O M900 e o M1000 demonstraram o início da formação de uma fase cristalina de difícil caracterização pela pequena intensidade dos picos. A sua identificação foi por meio do estudo in situ por DRX. O difratograma obtido a $970{ }^{\circ} \mathrm{C}$ (Fig. 2), com $45 \mathrm{~min}$, apresentou uma melhor definição dos picos da fase cristalina e confirmou a presença de mullita, com seus picos mais intensos em 3,40, 3,43 e 5,40 A. A identificação da mullita foi confirmada com o arquivo PDF 79-1452. Na maioria dos trabalhos científicos existem controvérsias sobre as fases formadas na faixa de temperatura entre $925{ }^{\circ} \mathrm{C}$ a $975{ }^{\circ} \mathrm{C}$. Em 1959 foi reportado que a reação exotérmica que ocorre nessa faixa é geralmente considerada uma rápida recristalização, sendo que alumina gama e mulita se formam nesse estágio [17]. Foi relatado em 1989 que existem três interpretações principais para a reação exotérmica nessa faixa de temperatura [18]: a) interferência de picos de pequena intensidade de mullita, b) diz respeito à formação de aluminagama e c) a própria nucleação de mullita, principalmente quando a partir da caulinitas "bem cristalizadas" devido a melhor organização da direção do eixo c.

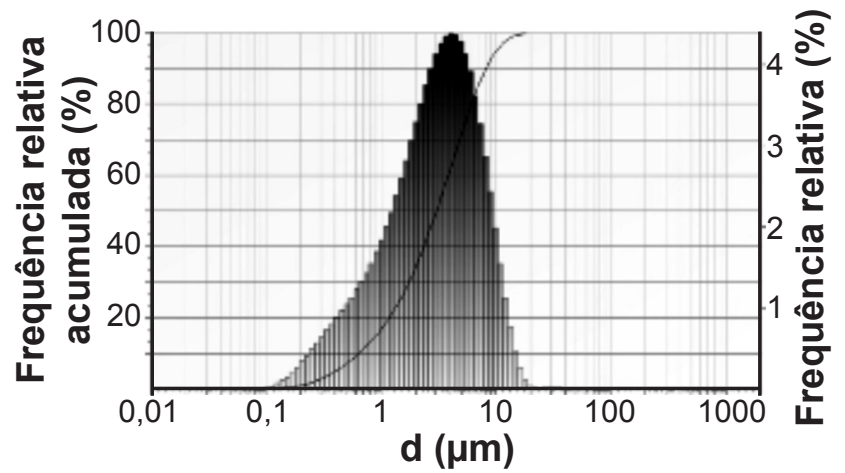

Figura 3: Distribuição granulométrica do caulim, d $(\mu \mathrm{m})$ é o diâmetro de partícula.

[Figure 3: Particle size distribution of kaolin, $d(\mu \mathrm{m})$ is the particle diameter.]

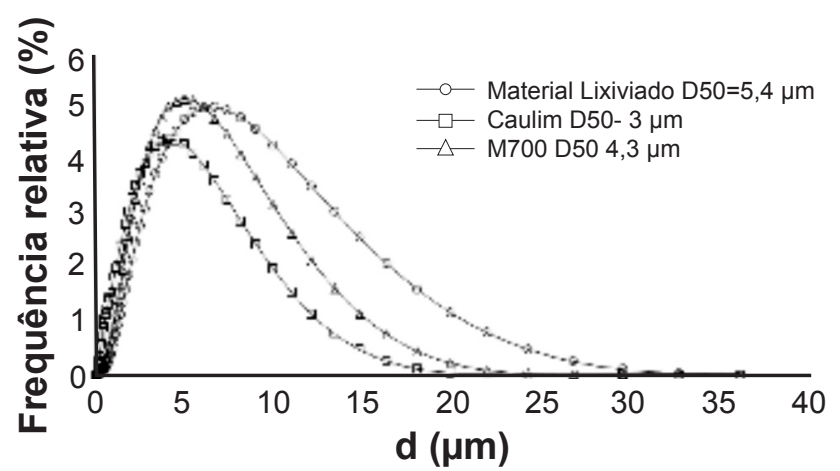

Figura 4: Distribuição granulométrica antes e depois da lixiviação, d $(\mu \mathrm{m})$ é o diâmetro de partícula $e \mathrm{D}_{50}$ é o diâmetro médio de partícula.

[Figure 4: Particle size distribution before and after leaching, $d$ $(\mu \mathrm{m})$ is the particle diameter and $D_{50}$ is the average of particle diameter.]

Tabela I - Composição química do caulim e metacaulim (\%). P.F.: Perda ao Fogo. [Table I - Chemical composition of kaolin and metakaolin (\%). P.F.: Loss of Ignition.]

\begin{tabular}{cccccccc}
\hline & $\mathrm{SiO}_{2}$ & $\mathrm{Al}_{2} \mathrm{O}_{3}$ & $\mathrm{Fe}_{2} \mathrm{O}_{3}$ & $\mathrm{Na}_{2} \mathrm{O}$ & $\mathrm{P}_{2} \mathrm{O}_{5}$ & $\mathrm{TiO}_{2}$ & P.F. \\
\hline Caulim & 46,64 & 37,75 & 0,53 & 0,23 & 0,11 & 0,39 & 14,33 \\
M600 & 52,01 & 42,12 & 0,66 & 0,34 & 0,10 & 0,52 & 4,17 \\
M700 & 53,36 & 43,58 & 0,58 & 0,33 & 0,13 & 0,57 & 1,47 \\
M800 & 53,59 & 44,11 & 0,71 & 0,32 & 0,13 & 0,53 & 0,62 \\
M900 & 53,76 & 44,35 & 0,58 & 0,33 & 0,11 & 0,53 & 0,53 \\
M1000 & 53,29 & 44,82 & 0,62 & 0,36 & 0,12 & 0,50 & 0,30 \\
\hline
\end{tabular}


O caulim usado no trabalho apresentou um diâmetro médio de partícula $\left(D_{50}\right) 3 \mu \mathrm{m}$ e uma freqüência relativa acumulada de mais de $60 \%$ abaixo de $4 \mu \mathrm{m}$ (Fig. 3). O processo de calcinação do caulim a $700{ }^{\circ} \mathrm{C}$ e lixiviação do
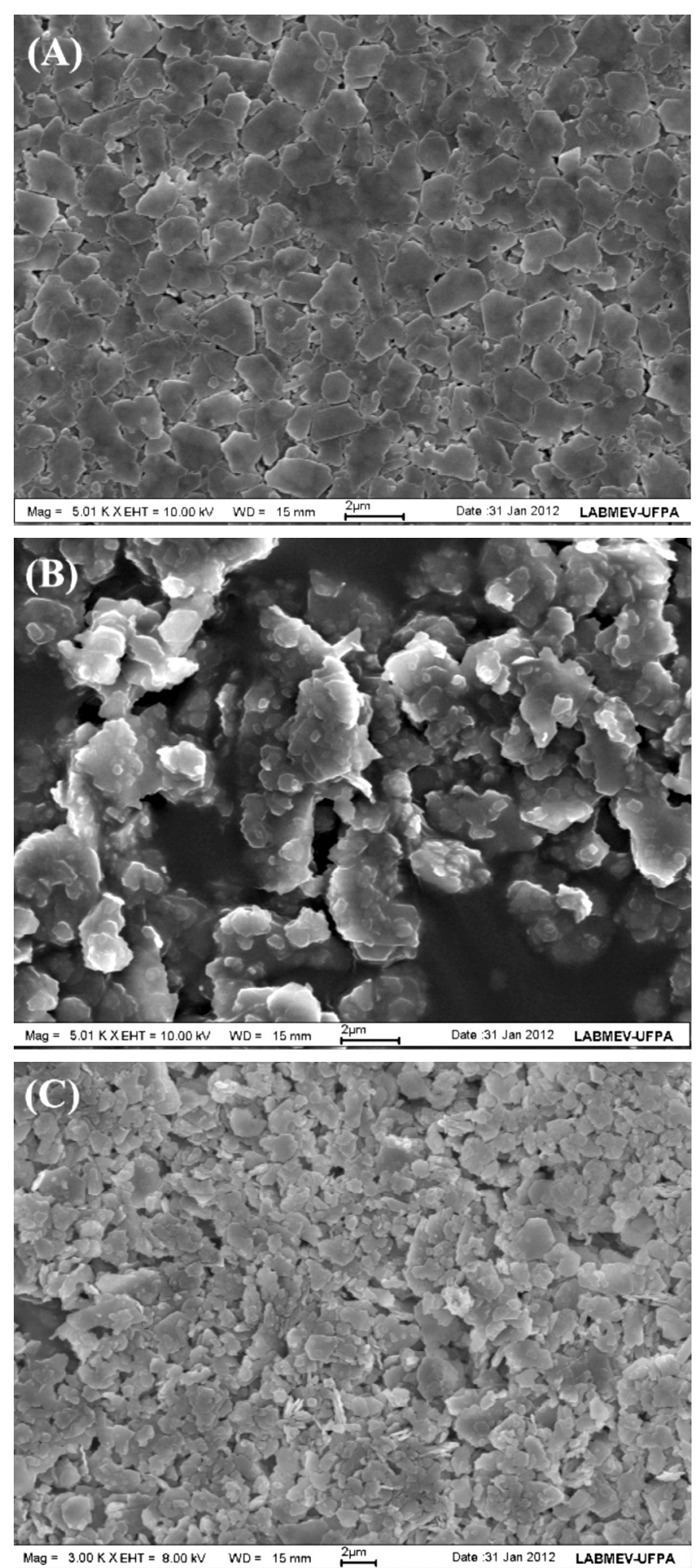

Figura 5: (A) Micrografias obtidas em microscópio eletrônico de varredura do caulim para cobertura, (B) M700 e (C) resíduo de sílica amorfa obtido após a lixiviação a $95{ }^{\circ} \mathrm{C}$.

[Figure 5: (A) SEM micrographs of kaolin for paper coating, (B) M700 and (C) amorphous silica residue obtained after leaching at $95{ }^{\circ} \mathrm{C}$.]
Tabela II - Área específica $\left(\mathrm{S}_{\mathrm{BET}}\right)$ antes e depois da lixiviação. [Table II - Specific area $\left(S_{B E T}\right)$ before and after leaching.]

\begin{tabular}{cccc}
\hline & Caulim & M700 & $\begin{array}{c}\text { Material } \\
\text { Lixiviado }\end{array}$ \\
\hline $\mathrm{S}_{\mathrm{BET}}\left(\mathrm{m}^{2} / \mathrm{g}\right)$ & 8,82 & 60,83 & 297,13 \\
\hline
\end{tabular}

mesmo a $95{ }^{\circ} \mathrm{C}$ não provocou alterações significativas na distribuição granulométrica dos materiais, pois os diâmetros médios do caulim, do M700 e do material lixiviado foram de 3, 4,3 e 5,4 $\mu \mathrm{m}$, respectivamente (Fig. 4). Os dados obtidos na análise da área específica para os mesmos materiais se encontram na Tabela II. Observouse que a calcinação do caulim a $700{ }^{\circ} \mathrm{C}$ elevou sua área específica e a lixiviação ácida a $95{ }^{\circ} \mathrm{C}$ por $3 \mathrm{~h}$ produziu sólidos com área de $297,13 \mathrm{~m}^{2} / \mathrm{g}$, referente à sílica amorfa formada durante o tratamento ácido. Segundo Ford [7] o aumento na área com o processo de lixiviação se deve ao crescimento do volume de micro e macro poros na estrutura do sólido. Em relação aos aspectos micro-morfológicos, na Fig. 5A foi possível observar as placas pseudo-hexagonais características da caulinita. Após a calcinação a $700{ }^{\circ} \mathrm{C}$ (Fig. 5B) e a lixiviação com ácido sulfúrico a $95^{\circ} \mathrm{C}$ (Fig. $5 \mathrm{C}$ ) verificou-se a permanência dessas placas.

\section{Lixiviação ácida}

As conversões fracionais obtidas nos processos de lixiviação com $\mathrm{H}_{2} \mathrm{SO}_{4}$ a $95{ }^{\circ} \mathrm{C}$ para o caulim, M700, M800, M900 e M1000 estão apresentadas na Fig. 6. Em 3 h de lixiviação a conversão para o M700 foi máxima, cerca de 0,9956, confirmando a sua alta reatividade, enquanto que a obtida para o M1000 foi o equivalente a 0,0659 , indicando a formação de um composto estável e com baixa reatividade. $\mathrm{O}$ valor final da conversão do alumínio para o caulim $(0,0842)$ foi ainda maior do que o obtido para o M1000 e diminuiu significativamente do M800 $(0,9165)$ para o M900 $(0,3506)$, cujos processos envolvidos se encontram em estudo. A quantificação da reatividade ou porcentagem de extração do alumínio do metacaulim foi realizada através da velocidade média de reação (Fig. 8). Foi considerado um tempo de 20 min de lixiviação e o valor obtido para o M700, neste tempo, foi dado como máximo $(100 \%)$ apenas para efeito de comparação. Com os dados encontrados (Tabela III) foi possível verificar que a reatividade do metacaulim diminuiu com o aumento da temperatura de calcinação e com redução mais significativa entre o M800 e o M900, sendo a reatividade do último a metade da encontrada para o M700 em um mesmo tempo. Como a reatividade máxima obtida foi doM700, o estudo cinético do processo de lixiviação foi realizado para este material nas temperaturas de $50,70,80$ e $95{ }^{\circ} \mathrm{C} \pm 3{ }^{\circ} \mathrm{C}$ conforme ilustrado na Fig. 7. Segundo Ford [7] a recuperação do alumínio é independente da granulometria do material analisado, é diretamente proporcional ao alumínio não-lixiviado presente, aumenta com a concentração do ácido e é extremamente dependente da temperatura. Este autor afirma ainda que um aumento 


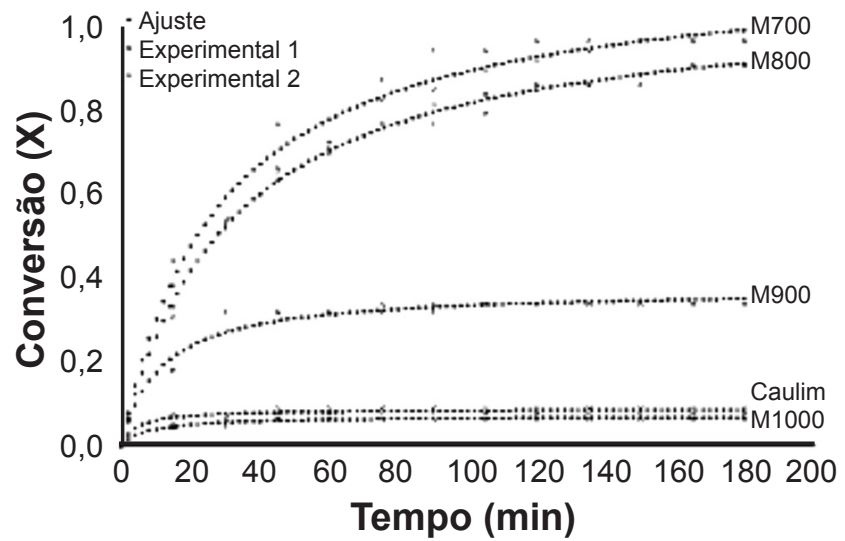

Figura 6: Conversão fracional (X) das lixiviações com $\mathrm{H}_{2} \mathrm{SO}_{4}$ a $95{ }^{\circ} \mathrm{C} \pm 3{ }^{\circ} \mathrm{C}$ para M700-1000.

[Figure 6: Fractional conversion $(X)$ of leachings with $\mathrm{H}_{2} \mathrm{SO}_{4}$ at $95{ }^{\circ} \mathrm{C} \pm 3{ }^{\circ} \mathrm{C}$ for $\mathrm{M700-1000.]}$

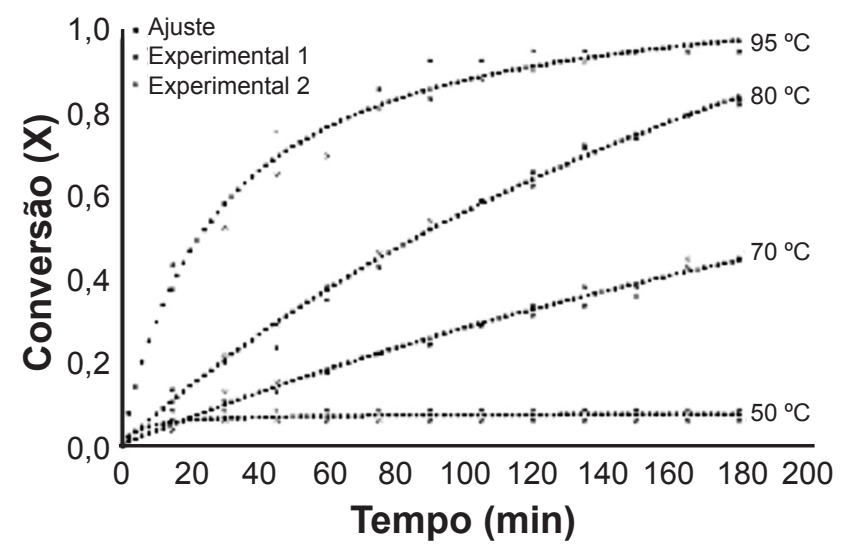

Figura 7: Conversão fracional (X) das lixiviações com $\mathrm{H}_{2} \mathrm{SO}_{4}$ a 50, 70,80 e $95^{\circ} \mathrm{C}$ para o M700.

[Figure 7: Fractional Conversion $(X)$ of leachings with $\mathrm{H}_{2} \mathrm{SO}_{4}$ at 50, 70, 80 and $95^{\circ} \mathrm{C}$ for the $\left.M 700.\right]$

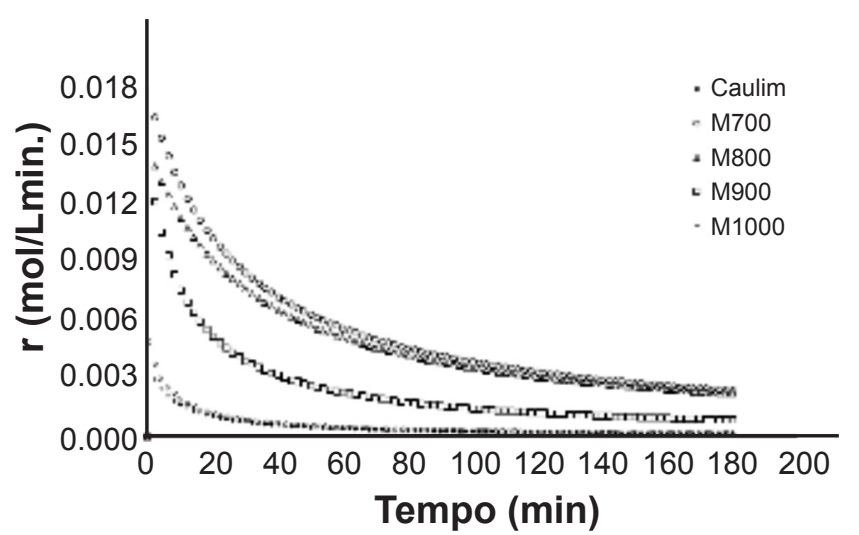

Figura 8: Velocidade média da reação (r) a $95^{\circ} \mathrm{C} \pm 3{ }^{\circ} \mathrm{C}$.

[Figure 8: Reaction rate average (r) at $95{ }^{\circ} \mathrm{C} \pm 3{ }^{\circ} \mathrm{C}$.]

da temperatura de $85{ }^{\circ} \mathrm{C}$ a $100{ }^{\circ} \mathrm{C}$ aumenta a dissolução do alumínio em $30 \%$ após $20 \mathrm{~min}$ e recuperações finais de alumínio de $87 \%$ a $85{ }^{\circ} \mathrm{C}$ e $97 \%$ a $100{ }^{\circ} \mathrm{C}$. Na presente pesquisa, o aumento da temperatura de $80{ }^{\circ} \mathrm{C}$ a $95{ }^{\circ} \mathrm{C}$ foi suficiente para aumentar a conversão em $30 \%$ e de $70{ }^{\circ} \mathrm{C}$ a
Tabela III - Reatividades dos materiais (\%). r: velocidade média de reação.

[Table III - Reactivity of the materials (\%). $r$ : reaction rate average.]

\begin{tabular}{ccc}
\hline Material & $\mathrm{r}$ (mol/Lmin.) & $\begin{array}{c}\text { Reatividade } \\
(\%)\end{array}$ \\
\hline M700 & 0,0102 & 100 \\
M800 & 0,0090 & 88 \\
M900 & 0,0052 & 51 \\
M1000 & 0,0011 & 11 \\
Caulim & 0,0013 & 13 \\
\hline
\end{tabular}

$95{ }^{\circ} \mathrm{C}$ ocorreu um acréscimo de $40 \%$ em um mesmo tempo de 20 min. Foram obtidas recuperações finais de alumínio, em termos de porcentagem de conversão, de $7 \%\left(50{ }^{\circ} \mathrm{C}\right)$, $45 \%\left(70{ }^{\circ} \mathrm{C}\right), 85 \%\left(80^{\circ} \mathrm{C}\right)$ e $99 \%\left(95^{\circ} \mathrm{C}\right)$. Devido ao baixo nível de conversão obtido a $50{ }^{\circ} \mathrm{C}$, seus dados não foram utilizados para o levantamento cinético.

\section{Estudo cinético}

Método integral: inicialmente propôs-se uma equação matemática da função da velocidade de reação, a qual se supôs que fosse aplicável aos dados que estavam sendo estudados:

$$
\text { Velocidade }=-\frac{\Delta\left[\mathrm{C}_{\mathrm{A}}\right]}{\Delta \mathrm{t}}=\mathrm{k}\left[\mathrm{C}_{\mathrm{A}}\right]
$$

$\mathrm{C}_{\mathrm{A}}$ é a concentração do reagente em um instante $\mathrm{t}$ e $\mathrm{k}$ é a constante de velocidade. Considerando a temperatura fixa e com uma única variável $\mathrm{C}_{\mathrm{A}}$, realizou-se a integração para obter a Equação Integrada da Velocidade:

$$
\operatorname{In} \frac{\left[\mathrm{C}_{\mathrm{A}}\right]_{\mathrm{t}}}{\Delta \mathrm{t}_{0}}=-\mathrm{kt}
$$

$\left[\mathrm{C}_{\mathrm{A}}\right]_{\mathrm{O}}$ é a concentração do reagente no instante $\mathrm{t}=0$ e $\left[\mathrm{C}_{\mathrm{A}}\right]_{\mathrm{t}}$ é a concentração no instante t. A partir da equação $D$ realizouse a aplicação dos procedimentos gráficos e o cálculo das constantes de velocidades. Segundo o método integral, o gráfico que se aplicou ao caso em questão corresponde ao do $\ln$ [concentração Inicial/ concentração] pelo tempo, de acordo com a equação $\mathrm{D}$, com desvio padrão $\left(\mathrm{R}^{2}\right)$ de 0,9915 , 0,9989 e 0,9999 para as lixiviações realizadas a 95, 80 e $70{ }^{\circ} \mathrm{C}$, respectivamente. Assim, através dos procedimentos gráficos, concluiu-se que a expressão da velocidade de reação proposta na equação $\mathrm{C}$ foi consistente com os dados cinéticos analisados, tratando-se de uma reação de primeira ordem em relação ao alumínio do metacaulim, de primeira ordem em relação à concentração de ácido sulfúrico e de segunda ordem global nas condições estudadas, de acordo com a equação E. As constantes de velocidades obtidas constam na Tabela IV.

$$
\mathrm{V}=\mathrm{k}\left[\mathrm{Al}_{2} \mathrm{Si}_{2} \mathrm{O}_{7}\right] \cdot\left[\mathrm{H}_{2} \mathrm{SO}_{4}\right]
$$




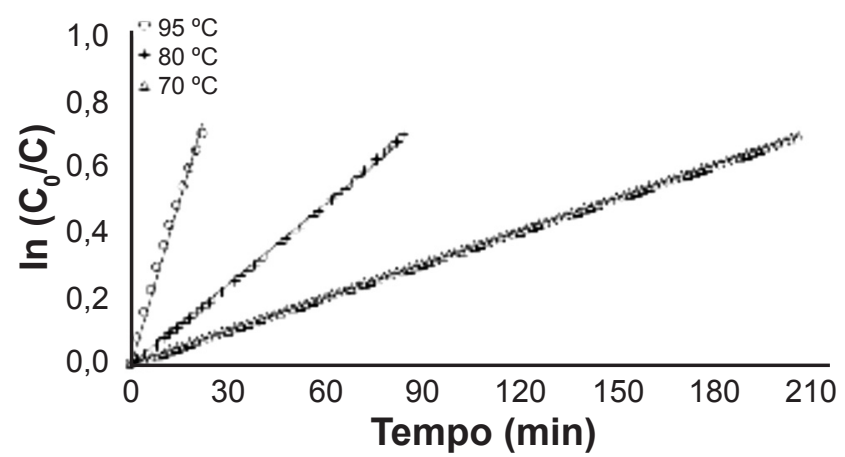

Figura 9: Análise da ordem da reação.

[Figure 9: Analysis of the reaction order.]

Tabela IV - Constantes de velocidade (k) obtidas para as temperaturas de lixiviação de 70,80 e $95^{\circ} \mathrm{C} \pm 3^{\circ} \mathrm{C}$.

[Table IV - Rate constants ( $k$ ) obtained for the leaching temperatures of 70,80 and $95{ }^{\circ} \mathrm{C} \pm 3{ }^{\circ} \mathrm{C}$.]

\begin{tabular}{cc}
\hline Temperatura $\left({ }^{\circ} \mathrm{C}\right)$ & $\mathrm{k}\left(\min \cdot{ }^{-1}\right)$ \\
\hline 95 & 0,0334 \\
80 & 0,0081 \\
70 & 0,0034 \\
\hline
\end{tabular}

Método das meias-vidas: foi aplicado de forma a confirmar a ordem de reação e as constantes de velocidades encontradas pelo método integral. Para um reagente com concentração $\mathrm{C}_{\mathrm{A}}$ numa reação de primeira ordem, a meiavida assume a seguinte forma:

$$
\left[\mathrm{C}_{\mathrm{A}}\right]_{\mathrm{t}}=\frac{1}{2}\left[\mathrm{C}_{\mathrm{A}}\right]_{0}
$$

$\left[\mathrm{C}_{\mathrm{A}}\right]_{\mathrm{O}}$ é a concentração inicial e $\left[\mathrm{C}_{\mathrm{A}}\right]_{\mathrm{t}}$ é a concentração depois de metade dos reagentes terem sido consumidos. Substituindo $\left[\mathrm{C}_{\mathrm{A}}\right]_{\mathrm{t}} /\left[\mathrm{C}_{\mathrm{A}}\right]_{\mathrm{o}}$ por $1 / 2$ e $\mathrm{t}$ pode $\mathrm{t}_{1 / 2}$ na Equação Integrada da Velocidade tem-se:

$$
\operatorname{In}\left(\frac{1}{2}\right)=-\mathrm{kt}_{1 / 2}
$$

Com isso, foi calculado o tempo de meia-vida $\left(\mathrm{t}_{1 / 2}\right)$ para as lixiviações realizadas com o M700 a 70, 80 e $95^{\circ} \mathrm{C}$, segundo as constantes de velocidades obtidas pelo método integral,e os resultados foram comparados com o tempo de meia-vida

Tabela V - Tempos de meia-vida obtidos para as lixiviações com M700 a 70,80 e $95{ }^{\circ} \mathrm{C} \pm 3{ }^{\circ} \mathrm{C}$.

[Table $V$ - Times of half-lives obtained for the leachings with $\mathrm{M700}$ at 70, 80 and $95^{\circ} \mathrm{C} \pm 3{ }^{\circ} \mathrm{C}$.]

\begin{tabular}{ccc}
\hline $\begin{array}{c}\text { Temperatura } \\
\left({ }^{\circ} \mathrm{C}\right)\end{array}$ & $\begin{array}{c}\mathrm{t}_{1 / 2} \text { Calculado } \\
(\mathrm{min} .)\end{array}$ & $\begin{array}{c}\mathrm{t}_{1 / 2} \text { Real } \\
(\mathrm{min} .)\end{array}$ \\
\hline 95 & 21 & 22 \\
80 & 86 & 84 \\
70 & 204 & 206 \\
\hline
\end{tabular}

real. A lixiviação promovida a $70{ }^{\circ} \mathrm{C}$ foi realizada durante um tempo de residência de $180 \mathrm{~min}$, logo não se obteve um tempo real para o consumo de metade dos reagentes iniciais. Assim, através da aplicação da equação obtida pelo ajuste dos pontos experimentais, estimou-se que o tempo de meia-vida seria em torno de $206 \mathrm{~min}$. O método meia-vida confirmou os resultados encontrados pelo método integral.

Método das velocidades iniciais: a velocidade de reação pode ser calculada, segundo o método, por diferenciação dos dados experimentais de concentração em função do tempo e posterior extrapolação do coeficiente angular para o tempo zero [15]. Considerando-se a expressão da velocidade de reação da seguinte forma:

$$
-\mathrm{r}_{0}=\mathrm{kC}_{\mathrm{A} 0}^{\alpha} \mathrm{C}_{\mathrm{B} 0}^{\beta}
$$

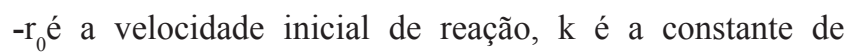
velocidade, $\mathrm{C}_{\mathrm{A} 0}$ é a concentração inicial do alumínio com ordem $\alpha$ e $\mathrm{C}_{\mathrm{B} 0}$ é a concentração inicial do $\mathrm{H}_{2} \mathrm{SO}_{4}$ com ordem $\beta$. A ordem da reação com relação a um componente individual, no caso $\mathrm{C}_{\mathrm{A}}^{\alpha}$, pode ser obtida através da realização de experimentos com concentrações iniciais diferentes do alumínio do metacaulim e mantendo a do $\mathrm{H}_{2} \mathrm{SO}_{4}$ constante. Assim, se denotarmos as duas velocidades observadas por $-\mathrm{r}_{0}$ e -r' ${ }_{0}$ nas concentrações iniciais $\mathrm{C}_{\mathrm{A} 0} \mathrm{e} \mathrm{C}_{\mathrm{A} 0}$, respectivamente, tem-se:

$$
\begin{aligned}
& \left(-r_{0}\right)=\left(k C_{B 0}^{\beta}\right) C_{A 0}^{\alpha} \\
& \left(-r_{0}\right)=\left(k C_{B 0}^{\beta}\right) C_{A 0}^{\alpha^{\prime}}
\end{aligned}
$$

Dividindo a equação I pela equação J, obtém-se:

$$
\begin{aligned}
& \frac{-r_{0}}{-r_{0}^{\prime}}=\left(\frac{C_{\mathrm{A} 0}}{C_{\mathrm{A} 0}^{\prime}}\right)^{\alpha} \\
& \alpha=\frac{\operatorname{In}\left(-r_{0} /-r_{0}\right)}{\operatorname{In}\left(C_{\mathrm{A} 0} /-C_{\mathrm{A} 0}^{\prime}\right)}
\end{aligned}
$$

Tabela VI- Velocidades de dissolução para as lixiviações realizadas com 0,21, 0,43 e 0,85M de alumínio (M700) e $1,5 \mathrm{M} \mathrm{H}_{2} \mathrm{SO}_{4}$ a $95 \pm 3{ }^{\circ} \mathrm{C}$. $-\mathrm{r}_{0}$ : velocidade de dissolução do alumínio.

[Table VI - Dissolution rates for the leachings carried out with 0.21, 0.43 and 0.85M aluminum (M700) and 1,5M $\mathrm{H}_{2} \mathrm{SO}_{4}$ at $95 \pm 3{ }^{\circ} \mathrm{C}$. $-\mathrm{rO}$ : dissolution rates of aluminum.]

\begin{tabular}{cccc}
\hline & \multicolumn{4}{c}{$\mathrm{C}_{\mathrm{A} 0}(\mathrm{~mol} / \mathrm{L})$} \\
$-\mathrm{r}_{0}(\mathrm{~mol} / \mathrm{L} . \mathrm{s}.) \times 10^{-4}-\mathrm{t}$ min. & 0,21 & 0,43 & 0,85 \\
\hline $15 \mathrm{~min}$. & 0,60 & 1,04 & 1,90 \\
$20 \mathrm{~min}$. & 0,46 & 0,89 & 1,70 \\
$25 \mathrm{~min}$. & 0,38 & 0,77 & 1,54 \\
$30 \mathrm{~min}$. & 0,32 & 0,68 & 1,40 \\
\hline
\end{tabular}


A partir da equação L e dos dados obtidos (Tabela VI) encontrou-se uma ordem de reação equivalente a $0,97 \mathrm{em}$ relação ao alumínio do metacaulim. A constante de velocidade foi calculada segundo a equação D a partir do coeficiente angular da reta ajustada do gráfico $\ln$ (concentração inicial/ concentração) em função do tempo. Foi encontrado um valor médio de $0,0342 \mathrm{~min}^{-1}$ que se encontra na mesma ordem de grandeza da obtida a $95{ }^{\circ} \mathrm{C}$ pelo método integral $\left(0,0334 \mathrm{~min}^{-1}\right)$.

Energia de ativação: a energia de ativação foi calculada segundo a equação de Arrhenius:

$$
\mathrm{k}=\mathrm{A} \mathrm{e}^{\frac{\mathrm{Ea}}{\mathrm{RT}}}
$$

$\mathrm{k}$ é a constante de velocidade da reação, A é o fator de frequência, $\mathrm{E}_{\mathrm{a}}$ é a energia de ativação $(\mathrm{J} / \mathrm{mol})$, $\mathrm{R}$ é a constante dos gases ideais e $\mathrm{T}$ é a temperatura da reação em K. Os resultados obtidos nos cálculos de energia de ativação se encontram na Tabela VII e plotados graficamente na Fig. 11, $\mathrm{T}$ é a temperatura absoluta.

Tabela VII - Dados para aplicação da equação de Arrhenius, $\mathrm{k}$ é a constante de velocidade.

[Table VII - Data for application of the Arrhenius equation, $k$ is the rate constant.]

\begin{tabular}{cccc}
\hline T. $(\mathrm{K})$ & $\mathrm{k}\left(\mathrm{s}^{-1}\right)$ & $(1 / \mathrm{T}) \mathrm{K}^{-1} \times 10^{4}$ & $-\operatorname{lnk}$ \\
\hline 343 & 0,00006 & 29,15 & 9,78 \\
353 & 0,00013 & 28,33 & 8,91 \\
368 & 0,00056 & 27,17 & 7,49 \\
\hline
\end{tabular}

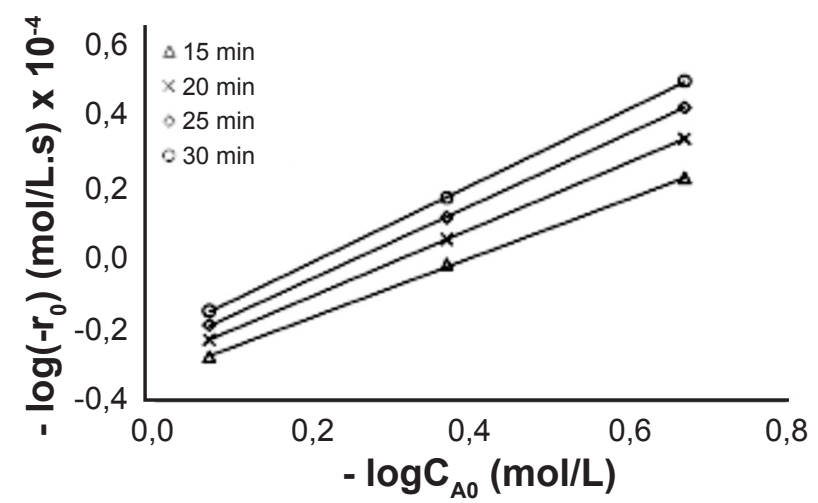

Figura 10: Velocidade inicial em função da concentração de alumínio.

[Figure 10: Initial rate as a function of initial aluminum concentration.]

Com um desvio padrão $\left(\mathrm{R}^{2}\right)$ de 0,9982 , a energia de ativação encontrada foi $96,25 \mathrm{~kJ} / \mathrm{mol}$. O cálculo da energia de ativação pode ser encontrado em alguns trabalhos sobre processos de lixiviação do alumínio utilizando caulim calcinado a $700-750{ }^{\circ} \mathrm{C}$, ácido sulfúrico com excesso superior a $90 \%$ e modelos de reação heterogênea. Por exemplo, Hulbert e Huff [6] compararam taxas de lixiviação do caulim calcinado em vários ácidos, testaram os dados com

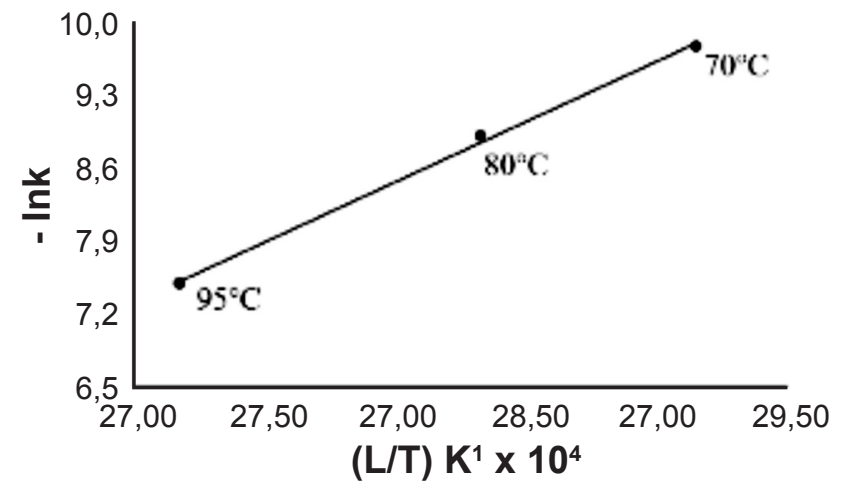

Figura 11: Procedimento gráfico para cálculo da energia de ativação.

[Figure 11: Graphic procedure for calculating activation energy.]

um modelo de reação heterogênea de nucleação, encontraram reação de primeira ordem em relação ao alumínio e energia de ativação equivalente a 121,4 kJ/mol para o ácido sulfúrico. Ford [7] utilizou o modelo do núcleo não reagido para o estudo cinético de um processo de lixiviação ácida de um caulim africano, tendo encontrado uma reação de lixiviação de primeira ordem em relação ao alumínio do metacaulim e energia de ativação de 67,4 kJ/mol. Altiokka, Akalin, Melek e Akyalçin [12] aplicaram o mesmo modelo utilizado por Ford no estudo da cinética de dissolução do metacaulim e encontraram uma reação de ordem 0,75 com respeito à concentração ácida e energia de ativação equivalente a $98,4 \mathrm{~kJ} / \mathrm{mol}$. Assim, a energia de ativação obtida no presente trabalho encontra-se na mesma ordem de grandeza das relatadas em trabalhos anteriores.

\section{CONCLUSÕES}

As curvas de velocidade média de reação demonstraram que o aumento da temperatura de calcinação do caulim diminuiu a reatividade do metacaulim obtido na faixa de temperatura de $700-1000^{\circ} \mathrm{C}$. O caulim amazônico calcinado a $700{ }^{\circ} \mathrm{C}$ por $2 \mathrm{~h}$ e lixiviado com ácido sulfúrico a $95 \pm 3{ }^{\circ} \mathrm{C}$ por $3 \mathrm{~h}$ apresentou máxima conversão de alumínio, cerca de $99,56 \%$. Segundo os métodos de reação homogênea aplicados, concluiu-se tratar de uma reação de primeira ordem em relação ao alumínio do metacaulim e de primeira ordem em relação à concentração do ácido sulfúrico. Foi encontrada uma energiade ativação equivalente a 96,25 $\mathrm{kJ} / \mathrm{mol}$. A distribuição granulométrica do caulim antes e depois do processo de lixiviação praticamente não sofreu variação, com diâmetro médio de partícula $\left(\mathrm{D}_{50}\right)$ para o caulim, M700 e material lixiviado equivalente a 3, 4,3 e 5,4 $\mu \mathrm{m}$, respectivamente. A área específica aumentou significativamente com este processo, passando de $8,82 \mathrm{~m}^{2} / \mathrm{g}$ (caulinita) para $297,13 \mathrm{~m}^{2} / \mathrm{g}$ no material calcinado a $700{ }^{\circ} \mathrm{C}$ e lixiviado a $95^{\circ} \mathrm{C}$. Finalmente, uma das principais contribuições deste trabalho, do ponto de vista de aplicação industrial, pode estar relacionada ao custo final do processo: a cinética de dissolução do metacaulim foi estudada com excesso de um dos reagentes de apenas $10 \%$ e métodos simples de reação homogênea, cujos resultados se encontram na mesma ordem 
de grandeza dos obtidos em diferentes estudos realizados sobre o tema, utilizando excesso elevado (acima de 90\%) e métodos de reação heterogênea.

\section{AGRADECIMENTOS}

À CAPES pela bolsa de mestrado da primeira autora e ao $\mathrm{CNPq}$ pela bolsa de produtividade em pesquisa do segundo autor (Proc. 304.566/2007-1). Recursos financeiros para a realização deste trabalho foram obtidos dos seguintes projetos: "MINOMAT - Dos Minerais aos Novos Materiais", Edital 02/2007, PRONEX (FAPESPA/CNPq), e "Desenvolvimento de Processo de Síntese de Zeólitas a partir de Rejeito de Caulim da Amazônia e Aplicações industriais",Edital MCT/CT-Mineral/VALE/CNPq 12/2009, Proc. 550.297/2010-3.

\section{REFERÊNCIAS}

[1] B. S. Carneiro, R. S. Angélica, T. Scheller, E. A. S. de Castro, R. F. Neves, Cerâmica 49 (2003) 237.

[2] A. A. B. Maia, E. Saldanha, R. S. Angélica, C. A. G. Souza, R. F. Neves, Cerâmica 53 (2007) 319.

[3] A. A. B. Maia, R. S. Angélica, R. F. Neves, Cerâmica 54 (2008) 345.

[4] S. P. A. Paz, R. S. Angélica, R. F. Neves, Química Nova 33, 3 (2010) 579.
[5] M. S. Barata, R. S. Angélica, Cerâmica 58 (2012) 36.

[6] S. F. Hulbert, D. E. Huff, J. Clay Miner. 8 (1970) 337.

[7] K. J. R. Ford, Hydrometallurgy 29 (1992) 109.

[8] C. V. Phillips, K. J. Wills, Hydrometallurgy 9 (1982) 15.

[9] S. Y. Gajam, S. Raghavan, Hydrometallurgy15, (1985) 143.

[10] C. Belver, M. A. B. Muñoz, M. A. Vicente, Chem. Mater 14, 5 (2002) 2033.

[11] M. R. Altiokka, H. L. Hoşgün, Hydrometallurgy 68 (2003) 77.

[12] M. R. Altiokka, H. Akalin, N. Melek, S. Akyalçin, Ind. Eng. Chem. Res. 49, 24 (2010) 12379.

[13] J. J. Rodriguez-Carvajal, FULLPROF 2000. Laboratoire Léon BrillouinSaclay, France (http://www.llb.cea.fr/ fullweb/ powder.htm.)

[14] H. S. Fogler, Elementos de Engenharia das Reações Químicas, 4ª ed., LTC Ed., Rio de Janeiro, RJ (2009) 201.

[15] B. I. Silveira, Cinética Química das Reações Homogêneas, $1^{a}$ Ed., Edgard Blücher, S. Paulo, SP (1996) 70.

[16] J. C. Kotz, P. Treichel, Química e reações químicas, $4^{\mathrm{a}}$ ed., LTC Ed., Rio de Janeiro, RJ (2002) 9.

[17] G. W. Brindley, M. Nakahira, J. Am. Ceram. Soc. 42, 7(1959) 311.

[18] P. S. Santos, Ciência e Tecnologia de Argilas. 2a Ed., Edgar Blücher, S. Paulo, SP (1989) 277.

(Rec. 07/05/2012, Rev. 26/08/2012, 29/08/2012, Ac. 10/11/2012) 